\title{
New Methods Providing High Degree Polynomials with Small Mahler Measure
}

\author{
G. Rhin and J.-M. Sac-Épée
}

\section{CONTENTS}

1. Introduction

2. A Statistical Method

3. A Minimization Method

4. Conclusion and Prospects

Acknowledgments

References
2000 AMS Subject Classification: Primary 12_04, 11Y40;

Keywords: Mahler measure, polynomials table, random drawings
In this work, we propose two new methods devoted to provide a large list of new polynomials with high degree and small Mahler measure. First, by statistical considerations, we augment Mossinghoff's list of polynomials with degree at most 180, and then we give a new list of such polynomials of degree up to 300 . The second idea is to perturb polynomials of Mossinghoff's list, and for higher degrees, of this new list, and to use them as initial polynomials for a minimization method, which converges to new polynomials with lower Mahler measure.

\section{INTRODUCTION}

Recall that if

$$
P(x)=\sum_{k=0}^{n} a_{k} x^{k}, a_{k} \in \mathbb{C} \text { and } a_{n} \neq 0,
$$

the Mahler measure of $P(x)$ is defined to be

$$
M(P)=\left|a_{n}\right| \prod_{k=1}^{n} \max \left(1,\left|\alpha_{k}\right|\right),
$$

where the $\alpha$ are the roots of $P$. Lehmer's problem is to know if, for any positive real number $\varepsilon$, there exists a monic polynomial with integer coefficients such that its Mahler measure lies between 1 and $1+\varepsilon$.

M. J. Mossinghoff gave a list of noncyclotomic and irreducible polynomials, with Mahler measure less than 1.3, and with degree at most 180 . His list was enriched later by P. Lisonek. Mossinghoff's algorithms consist in testing adjusted cyclotomic products, sparse polynomials, and all polynomials of a fixed degree with coefficients in $\{-1,0,+1\}$.

A careful examination of known polynomials motivated us to search for a relation between them, and we finally thought that interesting polynomials of degree $n$ could be searched in sets of polynomials given by a multinormal distribution, with mean polynomial $x^{n}+1$.

(c) A K Peters, Ltd. $1058-6458 / 2003 \$ 0.50$ per page Experimental Mathematics 12:4, page 457 
In the following section, we recall some facts about multinormal distributions, and we explain how we simulated random drawings of such polynomials.

\section{A STATISTICAL METHOD}

Aiming to produce a large number of polynomials with small Mahler measure, it is obviously crucial to have an efficient criterion to preselect polynomials whose Mahler measure is to be computed. Considering already known polynomials available, for example, in Mossinghoff's list, it appears that for each degree $n$, good candidates are polynomials that are "close" to $x^{n}+1$, in a sense we will specify below.

Recall that the vector $\mathbf{X}=\left(x_{1}, \ldots, x_{n}\right)$ has the multivariate normal distribution (or multinormal distribution), written $N(\mu, V)$, if its joint density function is

$$
f_{\mathbf{X}^{T}}=\frac{1}{\sqrt{(2 \pi)^{n} \operatorname{det}(V)}} \exp \left(-\frac{1}{2}(x-\mu)^{T} V^{-1}(x-\mu)\right),
$$

where $\mathrm{V}$ is a positive definite symmetric matrix.

The main result is that if $\mathbf{X} \sim N(\mu, V)$, then $\mathbb{E}[\mathbf{X}]=\mu$ (i.e., $\mathbb{E}\left[\mathbf{X}_{i}\right]=\mu_{i}$ for all $i$ ). Moreover, $V=\operatorname{Cov}\left[\mathbf{X}_{i}, \mathbf{X}_{j}\right]$ (see [Cartea 99]). According to A. Cartea ([Cartea 99]), another meaningful way of introducing multivariate normality is the following: The vector $\mathbf{X}=\left(\mathbf{X}_{1}, \ldots, \mathbf{X}_{n}\right)^{T}$ of random variables is said to have the multinormal distribution if for all $\mathbf{a}^{T}=\left(\mathbf{a}_{1}, \ldots, \mathbf{a}_{n}\right) \in \mathbb{R}^{n}$, one has that $\mathbf{x}^{T} \mathbf{a}=\mathbf{a}_{1} \mathbf{X}_{1}+\ldots+\mathbf{a}_{n} \mathbf{X}_{n}$ has a univariate normal distribution.

The covariance matrix $V$ used in our searches was constructed using heuristic considerations. We found that using a value of 0.22 for the variance of each $X_{i}$ produces good results. Full covariance matrices used in some of our searches are available on our web site: http://www.mmas.univ-metz.fr/ jmse/Recherche/TheodesNbres/Mahler.

Recall also that if the polynomial $P$ is not reciprocal, then $M(P) \geq \theta_{0}$, where $\theta_{0}=1.324717 \ldots$ is the smallest Pisot number. Then, we obviously deal with reciprocal polynomials. Generating monic reciprocal polynomials of degree $2 d$ subject to a multinormal distribution amounts to generating $d$-vectors subject to such a distribution. Then, we prepared a sampling of polynomials consistent with previous wishes. At first, we suppressed cyclotomic factors in each polynomial. If the resulting polynomial was irreducible, we computed its Mahler measure, otherwise we examined each of its factors.

This method allowed us to add new polynomials to Mossinghof's list, and also to produce many new polynomials up to degree 300. A list of these new polynomials is available from our web site in the file NewPolyUpDeg300.html.

A surprising result is that our statistical method produces exclusively polynomials whose Mahler measures lie in the neighbourhood of the known limit points of Mahler measures.

Recall that the Mahler measure of a two-variables polynomial $P(x, y)$ is defined by

$$
M(P(x, y))=\exp \left(\int_{0}^{1} \int_{0}^{1} \log |P(e(s), e(t))| d s d t\right),
$$

where $e(s)=\exp (2 \pi i s)$.

The smallest known limit points of Mahler measures arise from polynomials in two variables and are recalled below:

$$
\begin{aligned}
& M\left(x^{2}\left(y^{2}-1\right)+x\left(y^{3}-1\right)+y\left(y^{2}-1\right)\right)=1.255433 \ldots \\
& M\left(x^{2}+x\left(y^{2}+y+1\right)+y^{2}\right)=1.285734 \ldots \\
& M\left(x^{2} y(y+1)+x\left(y^{4}-y^{2}+1\right)\right. \\
& \left.\quad+y^{2}(y+1)\right)=1.309098 \ldots \\
& M\left(x^{2}\left(y^{3}-1\right)+x\left(y^{5}-1\right)+y^{2}\left(y^{3}-1\right)\right)=1.315692 \ldots .
\end{aligned}
$$

For example, values of Mahler measures we found for polynomials obtained at degree 242 are significant:

$$
\begin{array}{ll}
1.285717550280 & x^{242}-x^{179}+x^{121}-x^{63}+1 \\
1.286006968000 & x^{242}+x^{135}-x^{121}+x^{107}+1 \\
1.286084277301 & x^{242}+x^{137}-x^{121}+x^{105}+1 \\
1.286308220628 & x^{242}+x^{182}-x^{121}+x^{60}+1 \\
1.286410933712 & x^{242}-x^{177}-x^{121}-x^{65}+1 \\
1.309612811496 & x^{242}-x^{224}+x^{157}-x^{121}+x^{85} \\
& -x^{18}+1 \\
1.316822330028 & x^{242}-x^{234}+x^{226}+x^{137}-x^{129} \\
& +x^{121}-x^{113}+x^{105}+x^{16} \\
& -x^{8}+1 .
\end{array}
$$

We choose to give here only polynomials with small length, but numerous polynomials (with large length and small Mahler measure) can be found on our web site. For this specific degree, we did not find any polynomial whose Mahler measure lies in the neighbourhood of 1.255433 ..., but such polynomials were found at other degrees, for example, at degree 202:

$$
\begin{array}{ll}
1.254787222031 & x^{202}-x^{174}+x^{129}-x^{101}+x^{73} \\
& -x^{28}+1 \\
1.255701490987 & x^{202}+x^{185}-x^{118}-x^{101}-x^{84} \\
& +x^{17}+1 .
\end{array}
$$




\begin{tabular}{|c|c|c|c|}
\hline $\mathrm{M}(\mathrm{P})<1.27$ & $1.27 \leq \mathrm{M}(\mathrm{P}) \leq 1.29$ & $1.29<\mathrm{M}(\mathrm{P}) \leq 1.312$ & $1.312<\mathrm{M}(\mathrm{P})$ \\
\hline 11 & 251 & 34 & 38 \\
\hline
\end{tabular}

TABLE 1.

\begin{tabular}{|c|c|c|c|}
\hline Degree 174 & Degree 176 & Degree 178 & Degree 180 \\
\hline 1 & 2 & 7 & 9 \\
\hline
\end{tabular}

TABLE 2.

Moreover, to make the best possible use of our random drawings, our program is also endowed with a subroutine which examines each random polynomial whose Mahler measure is small: If this polynomial is irreducible, it is retained, while its prime factors are examined if it is not. This method provided new polynomials of high degrees, small Mahler measure, large length, and especially large coefficients (please consult our web site). In the following lines, we give two examples. Since the polynomials we deal with are reciprocal, we merely give half the coefficients of these polynomials:

$\begin{array}{lllllllllllll}190 & 1.285184607031 & 1 & -1 & 1 & -1 & 0 & 1 & \\ -2 & 2 & -2 & 1 & 1 & -2 & 3 & -3 & 2 & 0 & -2 & 3 \\ -4 & 3 & -1 & -1 & 3 & -4 & 4 & -2 & 0 & 2 & -4 & \\ 4 & -3 & 1 & 1 & -3 & 4 & -3 & 2 & 0 & -2 & 3 \\ -3 & 2 & -1 & -1 & 2 & -2 & 2 & -1 & 0 & 1 & -1 & 1 \\ -1 & 0 & 0 & 0 & 0 & 0 & 0 & 0 & 1 & -1 & 1 & -1 \\ 0 & 1 & -2 & 3 & -3 & 2 & 0 & -2 & 4 & -5 & 4 & -2 \\ -1 & 4 & -6 & 6 & -4 & 1 & 3 & -6 & 7 & -6 & 3 & \\ 1 & -5 & 7 & -7 & 5 & -1 & -3 & 6 & -7 & & & \end{array}$

$\begin{array}{lllllllllll}250 & 1.284974533200 & 1 & 1 & 1 & 1 & 0 & -1 & -2 & -2\end{array}$ $\begin{array}{lllllllllllll}-2 & -1 & 1 & 2 & 3 & 3 & 2 & 0 & -2 & -3 & -4 & -3 & -1\end{array}$ $\begin{array}{llllllllllllll}1 & 3 & 4 & 4 & 2 & 0 & -2 & -4 & -4 & -3 & -1 & 1 & 3 & 4\end{array}$ $\begin{array}{llllllllllllll}3 & 2 & 0 & -2 & -3 & -3 & -2 & -1 & 1 & 2 & 2 & 2 & 1 & 0\end{array}$ $\begin{array}{llllllllllllll}-1 & -1 & -1 & -1 & 0 & 0 & 0 & 0 & 0 & 0 & 0 & 1 & 1 & 1\end{array}$ $\begin{array}{lllllllllllll}1 & 0 & -1 & -2 & -2 & -2 & -1 & 1 & 2 & 3 & 3 & 2 & 0\end{array}$ $\begin{array}{llllllllllllll}-2 & -4 & -5 & -4 & -2 & 1 & 4 & 6 & 6 & 4 & 1 & -3 & -6 & -\end{array}$ $\begin{array}{lllllllllllll}7 & -6 & -3 & 1 & 5 & 7 & 7 & 5 & 1 & -3 & -6 & -7 & -6\end{array}$ $\begin{array}{llllllllllllll}-3 & 1 & 4 & 6 & 6 & 4 & 1 & -2 & -4 & -5 & -4 & -2 & 0 & 2\end{array}$ $\begin{array}{lllllllllll}3 & 3 & 2 & 1 & 0 & -1 & -1 & -1 & -1 & -1 & \text {. }\end{array}$

Please note the large coefficients of these polynomials.

In Table 1, we show the number of polynomials $P$ found in four specific intervals.

In Table 2, we summarize (by degree) the number of new polynomials of degree less than 180 lacking in Mossinghoff's list.

In the third section, we look for polynomials with small Mahler measure by using a completely different method based on a minimization algorithm.

\section{A MINIMIZATION METHOD}

In this section, we will present a method devoted to provide new polynomials with small Mahler measures by using a minimization algorithm. The aim of this algorithm is to give sequences of polynomials whose Mahler measures decrease. Given an initial reciprocal polynomial, we let each coefficient (one monomial and its reciprocal monomial change at the same time) $a_{i}$ grow from $a_{i}-1$ to $a_{i}+1$ (from $a_{i}-2$ to $a_{i}+2$ for small degrees, i.e., up to degree 40$)$. More precisely, for a given starting polynomial $P$ of degree $2 n$, we examine polynomials $P \pm x^{n}$ and $P \pm\left(x^{n-i}+x^{i}\right)$ for $0<i<n$, and similarly for the small degree case where adjusting by 2 is allowed. We test each obtained polynomial, and we keep the polynomial which has the smallest measure (see examples below). This polynomial is then the new initial polynomial, and we stop the process when this initial polynomial does not change upon applying the algorithm to it.

This method is fast and gives interesting results. Let us give some examples. For reasons of conciseness, we reproduce here only tests for some small degrees, but we tested this algorithm up to degree 300 . In all cases, we noted that very few loops are necessary. Let us run the program at degree 10 :

Initial polynomial:

$x^{10}+x^{9}+x^{8}+x^{7}-x^{6}-x^{5}-x^{4}+x^{3}+x^{2}+x+1$

Mahler measure: 1.960942

First output

$x^{10}+x^{9}-x^{8}+x^{7}-x^{6}-x^{5}-x^{4}+x^{3}-x^{2}+x+1$

Mahler measure: 1.926067

Second output

$x^{10}+x^{9}+x^{7}-x^{6}-x^{5}-x^{4}+x^{3}+x+1$

Mahler measure: 1.883774

Third output

$x^{10}+x^{9}-x^{7}-x^{6}-x^{5}-x^{4}-x^{3}+x+1$

Mahler measure: 1.176281

Note that other initial polynomials suit as well. For the same degree, we get, for example,

the sequence: 
Initial polynomial:

$x^{10}+x^{9}+x^{8}+x^{7}-3 * x^{6}-3 * x^{5}-3 * x^{4}+x^{3}+x^{2}+x+1$

Mahler measure: 3.626141

First output

$x^{10}+x^{9}+x^{8}-x^{7}-3 * x^{6}-3 * x^{5}-3 * x^{4}-x^{3}+x^{2}+x+1$

Mahler measure: 2.178217

Second output

$x^{10}+x^{9}+x^{8}-x^{7}-x^{6}-3 * x^{5}-x^{4}-x^{3}+x^{2}+x+1$

Mahler measure: 1.867224

Third output

$x^{10}+x^{9}+x^{8}-x^{7}-x^{6}-x^{5}-x^{4}-x^{3}+x^{2}+x+1$

Mahler measure: 1.731382

Fourth output

$x^{10}+x^{9}-x^{7}-x^{6}-x^{5}-x^{4}-x^{3}+x+1$

Mahler measure: 1.176281

Another example with a higher degree initial polynomial also gives an idea of the efficiency of the method.

Initial polynomial:

$x^{38}+x^{37}+x^{36}+x^{35}-x^{32}-x^{31}-x^{30}-x^{29}+x^{26}+x^{25}+$

$x^{24}+x^{23}-x^{20}+x^{19}-x^{18}+x^{15}+x^{14}+x^{13}+x^{12}-x^{9}-$

$x^{8}-x^{7}-x^{6}+x^{3}+x^{2}+x+1$

Mahler measure: 2.111413

First output

$x^{38}+x^{36}+x^{35}-x^{32}-x^{31}-x^{30}-x^{29}+x^{26}+x^{25}+x^{24}+$ $x^{23}-x^{20}+x^{19}-x^{18}+x^{15}+x^{14}+x^{13}+x^{12}-x^{9}-x^{8}-$ $x^{7}-x^{6}+x^{3}+x^{2}+1$

Mahler measure: 2.040529

Second output

$x^{38}+x^{36}+x^{35}-x^{32}-x^{31}-x^{30}-x^{29}+x^{26}+x^{25}+x^{24}+$ $x^{23}+x^{22}-x^{20}+x^{19}-x^{18}+x^{16}+x^{15}+x^{14}+x^{13}+x^{12}-$ $x^{9}-x^{8}-x^{7}-x^{6}+x^{3}+x^{2}+1$

Mahler measure: 2.002712

Third output

$x^{38}+x^{36}+x^{35}-x^{32}-x^{31}-x^{30}-x^{29}+x^{26}+x^{25}+x^{24}+$

$x^{23}+x^{22}-x^{20}-x^{19}-x^{18}+x^{16}+x^{15}+x^{14}+x^{13}+x^{12}-$

$x^{9}-x^{8}-x^{7}-x^{6}+x^{3}+x^{2}+1$

Mahler measure: 1.816100

Fourth output

$x^{38}+x^{36}+x^{35}-x^{32}-x^{31}-x^{30}-x^{29}+x^{26}+x^{25}+x^{24}+$ $x^{23}-x^{22}-x^{20}-x^{19}-x^{18}-x^{16}+x^{15}+x^{14}+x^{13}+x^{12}-$ $x^{9}-x^{8}-x^{7}-x^{6}+x^{3}+x^{2}+1$

Mahler measure: 1.686410

Fifth output

$x^{38}+x^{36}+x^{35}-x^{32}-x^{31}-x^{30}-x^{29}+x^{26}+x^{25}+x^{24}+$ $x^{23}-x^{20}-x^{19}-x^{18}+x^{15}+x^{14}+x^{13}+x^{12}-x^{9}-x^{8}-$ $x^{7}-x^{6}+x^{3}+x^{2}+1$

Mahler measure: 1.391285
Sixth output

$x^{38}+x^{37}+x^{36}+x^{35}-x^{32}-x^{31}-x^{30}-x^{29}+x^{26}+$ $x^{25}+x^{24}+x^{23}-x^{20}-x^{19}-x^{18}+x^{15}+x^{14}+x^{13}+$ $x^{12}-x^{9}-x^{8}-x^{7}-x^{6}+x^{3}+x^{2}+x+1$

Mahler measure: 1.268142

Of course, it is interesting to choose an initial polynomial that has already a small Mahler measure. For example, choosing $x^{188}+x^{165}-x^{94}+x^{23}+1$ with Mahler measure $1.286573 \ldots$ yields $x^{188}+x^{165}+x^{94}+x^{23}+1$, with Mahler measure $1.283092 \ldots$. However, the minimization method gives local minima, but not necessarily absolute minima, because the process stops every time a local minimum is found. Consequently, if the initial polynomial has a very small Mahler measure, this polynomial may be a minimum for the method. Therefore, we chose to take polynomials appearing in Mossinghoff's list and to perturb them to get good initial polynomials, and for higher degrees, we perturbed polynomials obtained with the statistical method of Section 2. More precisely, for producing starting polynomials, we took polynomials of Mossinghoff's list, or polynomials found with the statistical method, and we chose to change only the central monomial in the following way: If the coefficient of the central monomial is not 0 , we replace it by zero, while we replace it by 1 if it is equal to 0 .

Very promising for small degrees, the minimization method is at least less efficient than the statistical method. Actually, the ratio of the number of new polynomials found with the minimization method (for high degrees) compared with the number of new polynomials found with the statistical method is 1 to 10 . Moreover, the 19 new polynomials (with degree less than 180) lacking in Mossinghoff's list were found with the statistical method.

Finally, an interesting plan seems to test other minimization algorithms in order to increase the efficiency and speed of the minimization approach.

\section{CONCLUSION AND PROSPECTS}

To sum up, these two methods seem to be interesting approaches to discovering new polynomials with high degree and small Mahler measure, with a preference for the statistical method. In the near future, we plan to implement an automated version of the statistical method, which could perform indefinitely random drawings of polynomials and enrich the list each time a new interesting polynomial is found. We also plan to test various minimization algorithms to improve the method proposed in Section 
3. Finally, we undertook to study properties of polynomials with small Mahler measure from the angle of the localization of roots of such polynomials. That will be the theme of future work.

\section{ACKNOWLEDGMENTS}

The authors acknowledge sincerely the referee for very valuable and helpful advice and remarks.

\section{REFERENCES}

[Boyd 80] D. W. Boyd. "Reciprocal Polynomials Having Small Measure." Math. Comp. 35 (1980), 1361-1377.

[Boyd 81] D. W. Boyd. "Speculations Concerning the Range of Mahler's Measure." Canad. Math. Bull. 24 (1981), 453-469.

[Boyd 89] D. W. Boyd. "Reciprocal Polynomials Having Small Measure II." Math. Comp. 53 (1989), 355-357, S1S5.

G. Rhin, Laboratoire de Mathématiques de l'Université de Metz, Ile du Saulcy, 57045 Metz Cedex 01, France (rhin@poncelet.univ-metz.fr)

J.-M. Sac-Épée, Laboratoire de Mathématiques de l'Université de Metz, Ile du Saulcy, 57045 Metz Cedex 01, France (jmse@poncelet.univ-metz.fr)

Received June 25, 2002; accepted in revised form September 25, 2003.
[Cartea 99] A. Cartea. "Probability and Distribution Theory." Free course available from World Wide Web (http://www.econ.bbk.ac.uk/faculty/cartea/ ProbabilityandDistributions.pdf), 1999.

[Flammang et al. 97] V. Flammang, G. Rhin, and C. J. Smyth. "The Integer Transfinite Diameter of Intervals and Totally Real Algebraic Integers." J. Théor. Nombres Bordeaux 9 (1997), 137-168.

[Mossinghoff 98] M. J. Mossinghoff. "Polynomials with Small Mahler Measure." Math. Comp. 67 (1998), 1697-1705.

[Mossinghoff 02] M. J. Mossinghoff. "Small Salem Numbers." Available from World Wide Web (http://www.cecm. sfu.ca/ mjm/Lehmer/), 2002.

[Smyth 71] C. J. Smyth. "On the Product of the Conjugates Outside the Unit Circle of an Algebraic Integer." Bull. London. Math. Soc. 3 (1971), 169-175. 